\title{
Fully electronic urine dipstick probe for combinatorial detection of inflammatory biomarkers
}

\author{
Vikramshankar Kamakoti ${ }^{1}$, David Kinnamon ${ }^{1}$, Kang Hyeok Choi ${ }^{1}$, Badrinath Jagannath ${ }^{1}$ \& \\ Shalini Prasad*,1 \\ ${ }^{1}$ Department of Bioengineering, University of Texas at Dallas, 800 W Campbell Rd, Richardson, TX 75080, USA \\ *Author for correspondence: shalini.prasad@utdallas.edu
}

\begin{abstract}
Aim: An electrochemical urine dipstick probe biosensor has been demonstrated using molybdenum electrodes on nanoporous polyamide substrate for the quantitative detection of two inflammatory protein biomarkers, CRP and IL-6. Materials \& methods: The electrode interface was characterized using $\zeta$-potential and Fourier transform infrared spectroscopy. Detection of biomarkers was demonstrated by measuring impedance changes associated with the dose concentrations of the two biomarkers. A proof of feasibility of point-of-care implementation of the biosensor was demonstrated using a portable electronics platform. Results \& conclusion: Limit of detection of $1 \mathrm{pg} / \mathrm{ml}$ was achieved for CRP and IL-6 in human urine and synthetic urine buffers. The developed portable hardware demonstrated close correlation with benchtop equipment results.
\end{abstract}

Lay abstract: Early disease diagnosis helps in minimizing complications from various disease conditions. Quantitative readout of test results is useful in eliminating the error due to interpretation of output. In this paper, we have demonstrated the possibility of detection of two analytes in urine which holds promise to be developed as a home-based initial screening platform.

First draft submitted: 17 November 2017; Accepted for publication: 20 February 2018; Published online: 27 March 2018

Keywords: EIS • inflammatory protein biomarkers $\bullet$ molybdenum affinity biosensor $\bullet$ portable electronic interface $\bullet$ urine dipstick probe

Urine, being one of the most easily accessible fluids of the human body, is an intriguing medium to investigate chronic noninvasive health conditions and infectious diseases due to the presence of numerous biomarkers in its composition $[1,2]$. Conventional laboratory procedures for urinalysis require long turnaround time and sophisticated instrumentation, which is undesirable [3]. However, in comparison, urinary dipstick-based biosensors have the advantages of rapid reporting, ease of manufacturing and low cost, making them attractive for use. However, commercially, these predominantly rely on qualitative colorimetric reporting mechanisms [4-6], while the literature does introduce some promising prospects in urinary biosensors, summarized in Table 1 . The advancements reflected in these works have expanded the detection of identified traditional urinary biomarkers such as human chorionic gonadotropin (hCG), glucose and creatinine toward the detection of various other markers associated with health conditions such as liver and kidney function, sepsis, tuberculosis [7] and ZIKA virus [8-10]. The development of quantitative reporting of these versatile biomarkers is of significant interest for accurate interpretation of disease conditions in the human body.

Quantitative detection of inflammatory biomarkers in urine assists in the early diagnosis of certain disease conditions. The detection of inflammatory biomarkers such as IL-6 and C-reactive protein (CRP) in urine hold significance in the preliminary detection of chronic diseases [22] as well as bacterial infections [23-25]. Urinary tract infections (UTIs), with inflammation being one of the primary symptoms, are among the most common bacterial infections with an annual healthcare expenditure of $\$ 3.5$ billion in the USA [26,27]. The elevated levels of IL- 6 in urine hold critical significance in the diagnosis of UTIs [28]. IL-6 is synthesized by T cells, endothelial cells, 
Table 1. Summary of urine-based biosensors for analyte detection.

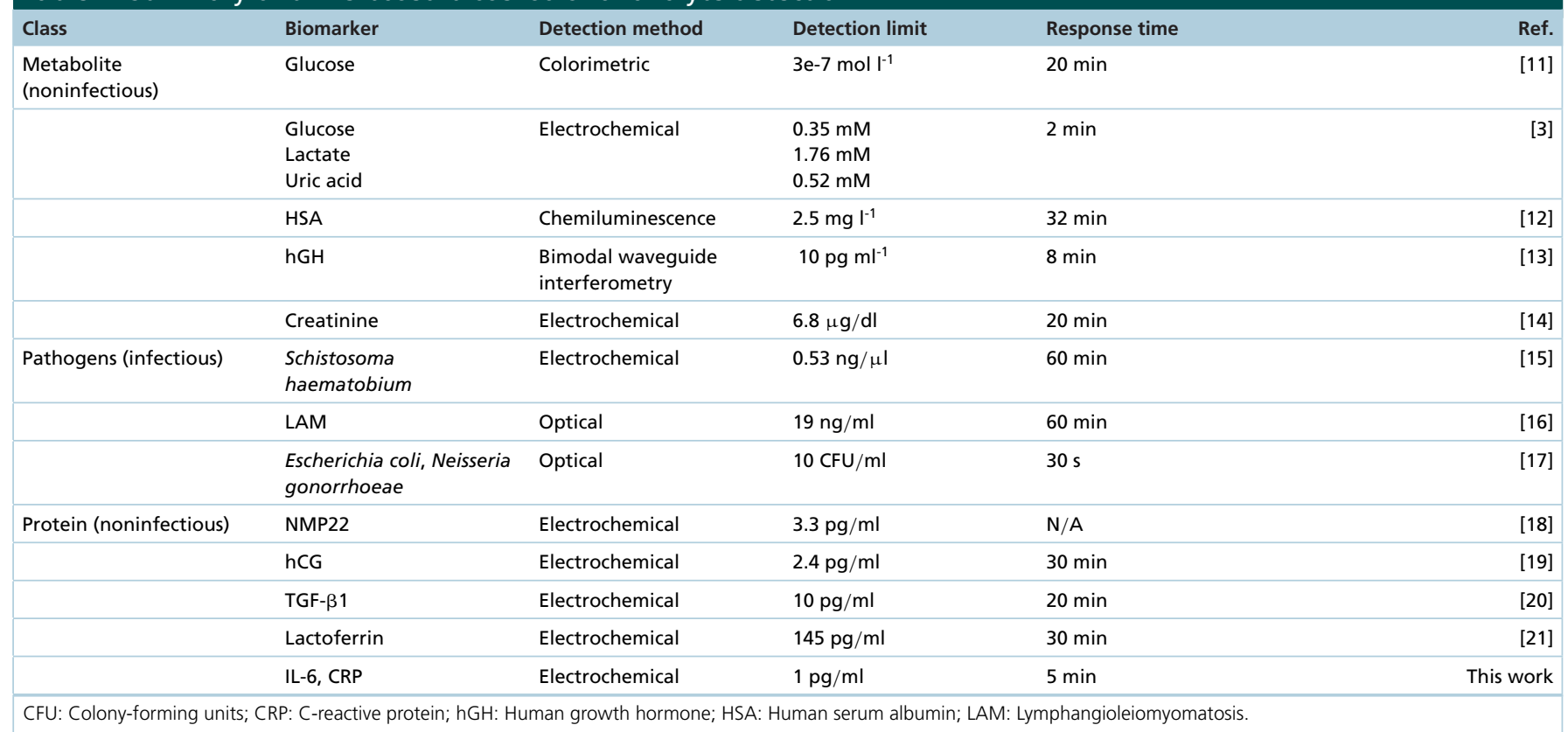

macrophages, B cells and fibroblasts after trauma and infection, resulting in inflammation. The local response of IL-6 occurs at the bladder mucosal surface as a response to bacterial infections [29]. The mean concentration of urinary IL-6 levels in healthy adults is approximately $3 \mathrm{pg} / \mathrm{ml}$ [30], while a concentration of $25 \mathrm{pg} / \mathrm{ml}$ is considered to be the threshold for requiring antibiotic treatment for UTIs [31]. Thus, detection of IL-6 in urine can serve as an initial screening tool aiding in the diagnosis of specific UTI infections.

The release of IL-6 triggers the liver to produce proteins such as CRP and fibrin. CRP is another key biomarker for diagnosis of inflammatory responses. It is recognized as a reliable method in differentiating bacterial and viral infections [32]. The mean concentration of urine CRP was established to be less than $150 \mathrm{pg} / \mathrm{ml}$ in healthy patients [33,34]. Both IL- 6 and hs-CRP have been shown to have clinical utility as biomarkers for diagnosing UTI $[35,36]$. Hence there may be an advantage in combinatorial detection of IL- 6 and CRP levels, as it would enable development of rapid point-of-care tests with a higher negative predictive value.

In this work, we present the development of a novel urinary diagnostic 'dipstick probe' biosensor, for combinatorial detection of inflammatory biomarkers: CRP and IL-6 through nonfaradaic electrochemical impedance spectroscopy (EIS). We leveraged the surface of a molybdenum (Mo) electrode on a flexible nanoporous polyamide substrate to develop an affinity assay for CRP and IL-6 detection using 1-ethyl-3-(3-dimethylaminopropyl) carbodiimide hydrochloride-N-hydroxysuccinimide (EDC-NHS) crosslinking chemistry. We have previously demonstrated the feasibility of Mo as a reliable electrode material for designing electrochemical biosensors [37]. The nanoscale network of intercalated pores of the polyamide membrane substrate allows for nanoconfinement of the target analyte enhancing the electrochemical response resulting from capacitive double-layer modulation [38]. The nanoporous structures also mitigate the charge screening effect caused by the nonspecific biomolecules [39]. The binding interactions of Mo with EDC-NHS crosslinker and antibodies were established using Fourier transform infrared spectroscopy (FTIR) and $\zeta$-potential characterization techniques. In this work, we propose a direct measure of specific urinary analyte through a fully electronic transduction method. We have established the detection capability of our biosensor in physiologically normal and elevated levels of IL- 6 and CRP in synthetic and human urine. At last, a proof-of-feasibility of a portable urinary dipstick probe electronic interface has been demonstrated. The aim of our work is to demonstrate the detection of inflammatory biomarkers in human urine for early disease detection. 


\section{Materials \& methods}

\section{Materials \& reagents}

NHS, EDC, 2-(N-Morpholino)ethanesulfonic acid (MES; pH 3) and phosphate-buffered saline (PBS; $0.1 \mathrm{M}, \mathrm{pH}$ 7) were obtained from Sigma-Aldrich (MO, USA). Anti-CRP antibody, anti-IL-6 antibody and CRP antigen were procured from Abcam (MA, USA). IL-6 protein was procured from Thermo Fisher Scientific, Inc. (MA, USA). Pooled human urine samples were purchased from Lee Biosolutions, Inc. (MO, USA). Acrylic cellulose acetate sheets were obtained from Staples (TX, USA). Kapton backing support material was obtained from McMaster Carr (TX, USA). Polyamide membranes of $200 \mathrm{~nm}$ pore size were obtained from GE Healthcare (NJ, USA). All solvents and reagents were of analytical grade and used as received.

\section{Fabrication \& surface characterization of Mo electrode}

The electrode pattern was deposited onto the base polyamide substrate using a shadow mask thin-film electronbeam deposition process. The shadow mask stencils were made from acrylic cellulose acetate polymer sheets. A thin base layer of gold was deposited below the Mo to improve electrode adhesion. A secondary acrylic adhesive glue was patterned to prevent fluid wicking on to contact pads of the electrodes. The deposition profile of the deposited Mo was evaluated using scanning electron microscopy (SEM), energy dispersive $\mathrm{x}$-ray spectroscopy (EDAX) and atomic force microscopy (AFM).

\section{FTIR characterization of surface chemistry}

Attenuated total reflectance Fourier transform infrared (ATR-FTIR) spectrograms were performed to establish the binding of the EDC-NHS crosslinker to the Mo surface, and subsequent binding of IL-6 and CRP antibody to the EDC-NHS crosslinker. The tool was equipped with a deuterated triglycine sulfate detector and $\mathrm{KBr}$ window. A Harrick VariGATR sampling stage with a $65^{\circ}$ germanium ATR crystal was used in this study. Measurements were taken as an average of 512 scans in the range of $4000-600 \mathrm{~cm}^{-1}$ with $4 \mathrm{~cm}^{-1}$ resolution. ATR-FTIR slides were prepared on cleaned glass slides with analogous metal deposition to the electrode fabrication process. The slides were incubated with $100 \mu \mathrm{l}$ of the EDC-NHS mixture for $1 \mathrm{~h}$. A $3 \times$ PBS wash was performed to remove any unbound crosslinker. After washing and completely drying with nitrogen, the Mo EDC-NHS measurement was taken. After EDC-NHS functionalization, $100 \mu \mathrm{l}$ of $10 \mu \mathrm{g} / \mathrm{ml}$ target antibody was incubated on the slide surface for an additional $30 \mathrm{~min}$ (one slide for IL-6 antibody and one slide for CRP). A $3 \times$ PBS wash was performed to remove unbound antibodies and the slides were dried with nitrogen and silica desiccant beads before the measurements were taken. All measurements represent individual slide preparations, with none being used more than once.

\section{Surface charge characterization with $\zeta$-potential}

$\zeta$-potential measurements were performed to validate the construction of the immunoassay onto the Mo surface using Malvern Instrument's Zetasizer Nano ZS (MA, USA). Electrophoretic mobility technique was used for $\zeta$ potential measurements. First, $\zeta$-potential of Mo (VI) oxide nanoparticles (Sigma-Aldrich) was measured followed by EDC-NHS bound to the Mo nanoparticles. Further, $\zeta$-potential of anti-IL- 6 and anti-CRP antibodies bound to Mo EDC-NHS complex was measured. $1 \mathrm{ml}$ of sample volume was used for each measurement. $\zeta$-potential for IL- 6 and CRP dose concentrations was performed in triplicate to validate the binding of immunoassay in synthetic urine at $\mathrm{pH} 6.45$ between $10 \mathrm{pg} / \mathrm{ml}$ and $100 \mathrm{ng} / \mathrm{ml}$ using the assay developed on Mo surface. Smoluchowski approximation was used to compute the $\zeta$-potential as given below:

$$
\mu=\frac{\varepsilon . \zeta}{\eta}
$$

$\mu$ : Electrophoretic mobility; $\varepsilon$ : Dielectric constant; $\zeta: \zeta$-potential; $\eta$ : Viscosity of solution.

\section{Experimental protocol for biosensing experiments}

A solution of $100 \mathrm{mM}$ NHS and $400 \mathrm{mM}$ EDC was dissolved in MES ( $\mathrm{pH}$ 3) buffer just prior to incubation on the sensor. The resulting EDC-NHS complex was then incubated on the sensor surface for $1 \mathrm{~h}$. Post-EDCNHS functionalization, $8 \mu \mathrm{l}$ of PBS was added to the sensing region to remove any unbound crosslinker. $8 \mu \mathrm{l}$ of $10 \mu \mathrm{g} / \mathrm{ml}$ of monoclonal antibody (IL-6 or CRP) in $0.1 \mathrm{M} \mathrm{PBS}$ was incubated for $15 \mathrm{~min}$. To emulate a true testing 
environment, test samples were prepared in spectrometer cuvettes containing $300 \mu \mathrm{l}$ of sample, which is sufficient to fully cover the sensor when they are dipped in the cuvette. After approximately $15 \mathrm{~s}$ of being continuously dipped in the sample, the sensor was removed from the cuvette and allowed to incubate for $5 \mathrm{~min}$. Studies were conducted in both synthetic urine and pooled human urine. The stability of Mo electrode to function efficiently in varying $\mathrm{pH}$ of urine was evaluated by performing electrochemical characterization studies in synthetic urine formulated with at $\mathrm{pH}$ 5.5, 6.5 and 7.5. A zero-dose measurement in the absence of target molecule was performed before introducing any samples. All dose concentrations of one target protein (either IL-6 or CRP) were tested on a sensor. IL- 6 was tested in the range of $1 \mathrm{pg} / \mathrm{ml}-10 \mathrm{ng} / \mathrm{ml}$, while CRP was tested in the range of $1 \mathrm{pg} / \mathrm{ml}-100 \mathrm{ng} / \mathrm{ml}$ mapping to their physiologically relevant ranges. EIS measurements were performed at each assay step on Gamry Reference 3000 potentiostat (PA, USA) and developed portable electronic reader in parallel. An AC voltage of $10 \mathrm{mV}_{\mathrm{RMS}}$ was applied with a frequency sweep of $1 \mathrm{~Hz}-1 \mathrm{MHz}$.

\section{Portable electronic dipstick interface}

The developed electronic reader represented in Figure $5 \mathrm{C}$ and $\mathrm{D}$ demonstrated the translatability of the biosensor to a portable form factor. The device was constructed in its entirety from off-shelf components, and was programed using open-source code libraries on Arduino Uno R3 microcontroller. The sinusoidal excitation waveform was generated using an MCP4725 digital-to-analog converter. The current response at the working electrode was captured using a current-to-voltage amplification circuit (INA 121) and read back into the microcontroller using an ADS1015 analog-to-digital converter. The sensor consumes no more than $5 \mu \mathrm{A}$ of current in a given measurement, and only takes $8-10 s$ to complete a measurement, making it an ideal transduction method for ultralow power biosensing.

\section{Results}

Surface characterization of Mo biosensor

A two-electrode electrochemical sensor was developed on porous polyamide (PA) substrate. The two-electrode system consists of a reference electrode which is curved around a circular working electrode at the center of the sensing region. The total area of the electrode was $0.7 \mathrm{~cm}^{2}$. The two electrodes were interfaced with test equipment using thin traces so that the sensing region of the electrode geometry dominates the impedance response. A COMSOL model of this electrode design included in Supplementary Figure 1, shows maximum distribution of current density and electric displacement field near the working electrode which is favorable for evaluating the current response from electrochemical characterization experiments [40].

Further, surface characterization of the Mo-deposited electrodes was performed using SEM and AFM techniques to establish the uniformity of the metal deposition. Figure 1C shows the SEM micrograph of the Mo-deposited PA substrate, while Figure 1D shows the EDAX characterization of the represented micrograph. The results depict a strong presence of Mo as evidenced by peaks at $2.29 \mathrm{keV}$. The resistivity of the deposited Mo electrode measured with four-point probe source meter was $332 \times 10^{-3} \Omega \mathrm{m}$. To establish the uniformity of the deposition, AFM characterization (Figure $1 \mathrm{~B}$ ) was used. A low root mean square $\left(\mathrm{R}_{\mathrm{q}}\right)$ roughness of $2.36 \mathrm{~nm}$ was measured, with $\mathrm{R}_{\mathrm{q}}$ value being an indicator of surface smoothness. With an $\mathrm{Rq}$ value of blank glass substrate being measured as $1.08 \mathrm{~nm}$, change in the surface roughness after Mo deposition can be considered negligible. The baseline electrochemical characterization of Mo electrode was performed through open-circuit potential experiments in synthetic urine buffer. Mo electrode demonstrated a stable open-circuit potential for $10 \mathrm{~min}$ (data not shown) of measurement indicating the stability of the electrode in the absence of any applied input voltage.

\section{ATR-FTIR surface characterization analysis}

ATR-FTIR was used to evaluate the nature of the chemical bonds present at the surface of the Mo electrode to validate the binding of both the EDC-NHS complex to the Mo surface as well as the subsequent binding of antibodies. Figure $2 \mathrm{~A}$ shows a visualization of the three measurements taken to characterize the functionalization steps for each antibody. Figure $2 \mathrm{~B}$ shows the resulting spectra. Table 2 outlines the associated wave number location for the relevant peaks in the figure. First, a measurement on blank Mo was done to characterize the presence of the Mo-native oxide, and to have a point of comparison for subsequent binding to the oxide surface. Next, the spectra postfunctionalization with the EDC-NHS crosslinker complex was taken, followed by measurements after the subsequent binding of IL- 6 or CRP antibody. The spectra of the postcrosslinker and postantibody presented in Figure $2 \mathrm{~A}$ and $\mathrm{C}$ are the result of subtraction from the Mo baseline to better visualize the peaks. The bare Mo sample shows an absence of discernible peaks with the exception of a broad peak manifesting from 900 to $1100 \mathrm{~cm}^{-1}$. This 

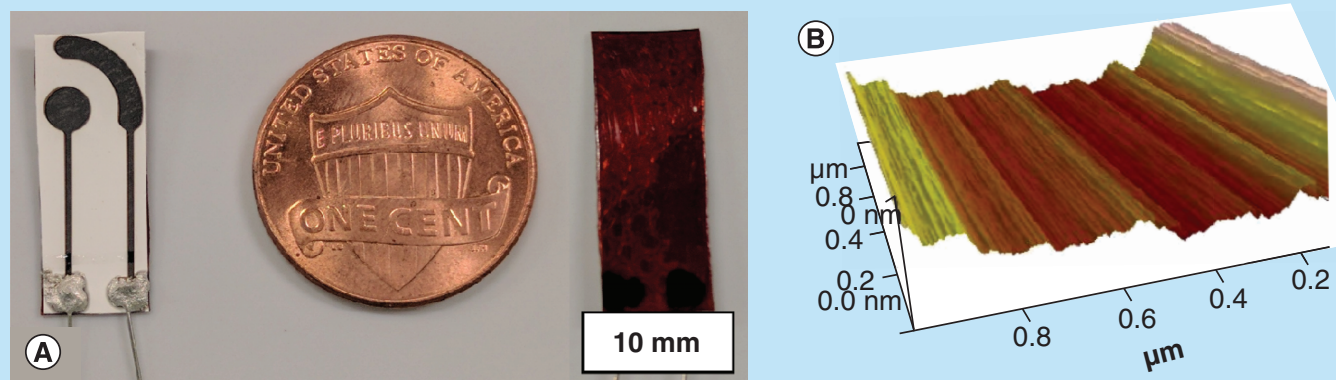

(D)
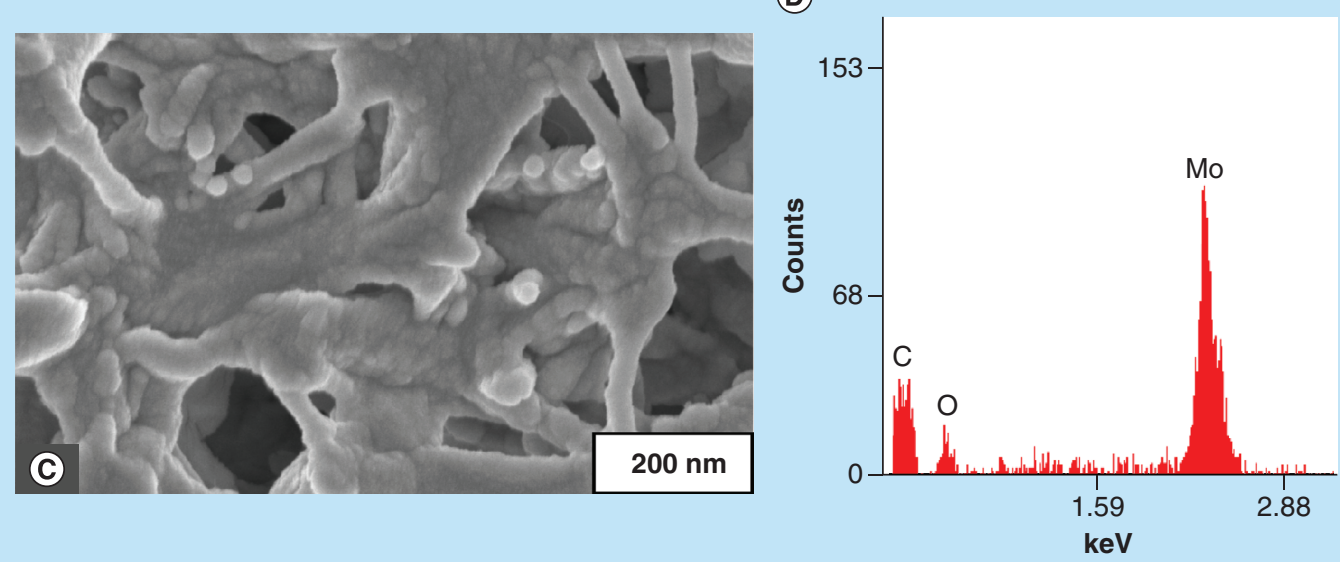

Figure 1. Surface characterization of molybdenum biosensor. (A) Two-electrode Mo biosensor with polyimide backing; (B) Atomic force microscopy image indicating uniform deposition profile of molybdenum; (C) scanning electron microscopy image indicating conformal deposition of molybdenum on polyamide substrate; (D) Energy dispersive $\mathrm{x}$-ray spectroscopy characterization of scanning electron microscopy region in (C).

Mo: Molybdenum.

Table 2. Fourier transform infrared spectroscopy characterization study of molybdenum and signature peak analysis.

\begin{tabular}{|c|c|c|c|c|}
\hline \multirow[t]{2}{*}{ Assignments } & \multicolumn{4}{|c|}{ Peak positions $\left(\mathrm{cm}^{-1}\right)$} \\
\hline & Mo & Mo + EDC-NHS & $\mathrm{Mo}+$ EDC-NHS + $\alpha$-IL- 6 & $\mathrm{Mo}+$ EDC-NHS + $\alpha-$ CRP \\
\hline$v(C=0)$ & $\mathrm{N} / \mathrm{A}$ & 1650 & 1647 & 1656 \\
\hline $\mathrm{V}_{\text {as }}(\mathrm{C}-\mathrm{N}, \mathrm{C}-\mathrm{C})$ & N/A & N/A & 1082 & 1047 \\
\hline
\end{tabular}

CRP: C-reactive protein; EDC: 1-ethyl-3-(3-dimethylaminopropyl) carbodiimide hydrochloride; Mo: Molybdenum; NHS: N-hydroxysuccinimide.

broad peak is characteristic of the Mo oxide layer that is leveraged for EDC-NHS binding. The FTIR spectra of the crosslinker and the antibody $(\mathrm{Ab})$-functionalized surfaces demonstrated signature peaks corresponding to the organic functional groups present in the respective molecules.

\section{Surface charge characterization using $\zeta$-potential}

$\zeta$-potential measurements can be used to quantify the modulation of surface charge due to the conjugation of charged species. Figure 2D shows that the $\zeta$-potential of Mo nanoparticles in deionized water is $-9.28 \mathrm{mV}$. The $\zeta$-potential decreases to $-5.01 \mathrm{mV}$ due to the binding of EDC-NHS to Mo nanoparticles resulting in a change in surface charge. The surface charge modulation due to the binding of antibody-antigen results in the modulation of electrical double layer (EDL) [41].

For the CRP affinity assay, the $\zeta$-potential after antibody conjugation is $-3.73 \mathrm{mV}$ as seen in Figure $2 \mathrm{D}$. Figure $2 \mathrm{E}$ shows that the $\zeta$-potential for three dose concentrations of CRP increases in magnitude from -1.24 to $-3.35 \mathrm{mV}$ for 

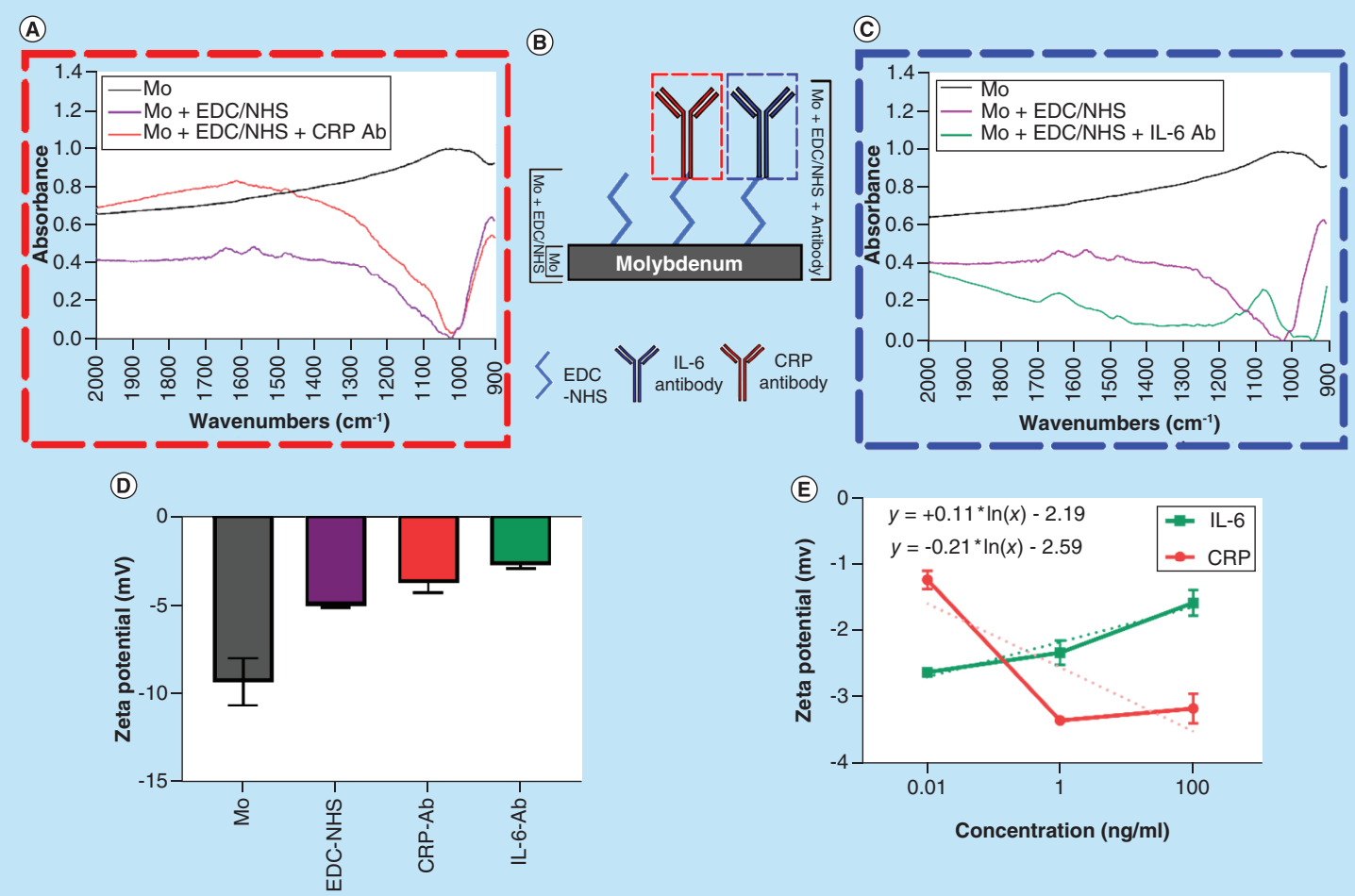

Figure 2. Surface characterization of functionalized molybdenum sample. (A) Fourier transform infrared spectroscopy (FTIR) spectra for functionalization of CRP; (B) Schematic representation of presented FTIR spectra; (C) FTIR spectra for functionalization of IL-6; (D) $\zeta$-potential measurements for CRP and IL- 6 antibodies; (E) $\zeta$-potential characterization of CRP and IL- 6 antigen concentrations. Ab: Antibody; CRP: C-reactive protein; EDC: 1-ethyl-3-(3-dimethylaminopropyl) carbodiimide hydrochloride; Mo: Molybdenum; NHS: N-hydroxysuccinimide.

dose concentration of $10 \mathrm{pg} / \mathrm{ml}$ and $1 \mathrm{ng} / \mathrm{ml}$, respectively. The slight drop in $\zeta$-potential to $-3.17 \mathrm{mV}$ at $100 \mathrm{ng} / \mathrm{ml}$ of CRP may be attributed to the saturation of CRP. For the IL- 6 affinity assay, the $\zeta$-potential after antibody conjugation is $-2.76 \mathrm{mV}$ as seen in Figure 2D. Figure $2 \mathrm{E}$ shows that the $\zeta$-potential for three dose concentrations of IL-6 decreases in magnitude from -2.6 to $-1.5 \mathrm{mV}$ with respect to increasing dose. This opposite behavior of CRP and IL- 6 due to the varied modulation of EDL with varying dose concentrations is also seen in EIS measurements (discussed in 'EIS characterization of CRP \& IL-6').

\section{EIS characterization of CRP \& IL-6}

EIS measurements were performed to electrochemically characterize the biosensing performance of the developed urine dipstick biosensor developed to detect CRP and IL-6 in urine.

\section{EIS characterization of CRP \& IL-6 in synthetic urine}

When testing physiological buffers such as urine, it is important to first characterize the impact performance of the sensor under various physiological conditions such as $\mathrm{pH}$ variation. Urine is of variable $\mathrm{pH}$ across patients and hence, any affinity biosensor that is sampling for biomarkers in urine must be robust for biosensing over physiological range of urine. EIS biosensing experiments were performed on the developed Mo electrodes for CRP and IL- 6 spiked in synthetic urine formulated according to the procedure described by $\mathrm{Xu}$ et al. [42] for $\mathrm{pH} 5.5$, 6.45 and 7.5 .

Figure $3 \mathrm{~A}$ represents the percentage change in impedance at $1 \mathrm{~Hz}$ frequency for low $(1 \mathrm{pg} / \mathrm{ml})$ and high $(100 \mathrm{ng} / \mathrm{ml})$ CRP concentrations spiked in synthetic urine of $\mathrm{pH}$ 5.5, 6.5 and 7.5. A decreasing trend in impedance change was observed for increasing dose concentrations of CRP, approximately $-20 \%$ and approximately $-60 \%$ for $1 \mathrm{pg} / \mathrm{ml}$ and $100 \mathrm{ng} / \mathrm{ml}$, respectively, across the $\mathrm{pH}$ ranges tested. The percentage change in impedance for low and the high CRP dose concentrations demonstrated statistical significance of $\mathrm{p}<0.05$ at $95 \%$ confidence interval 

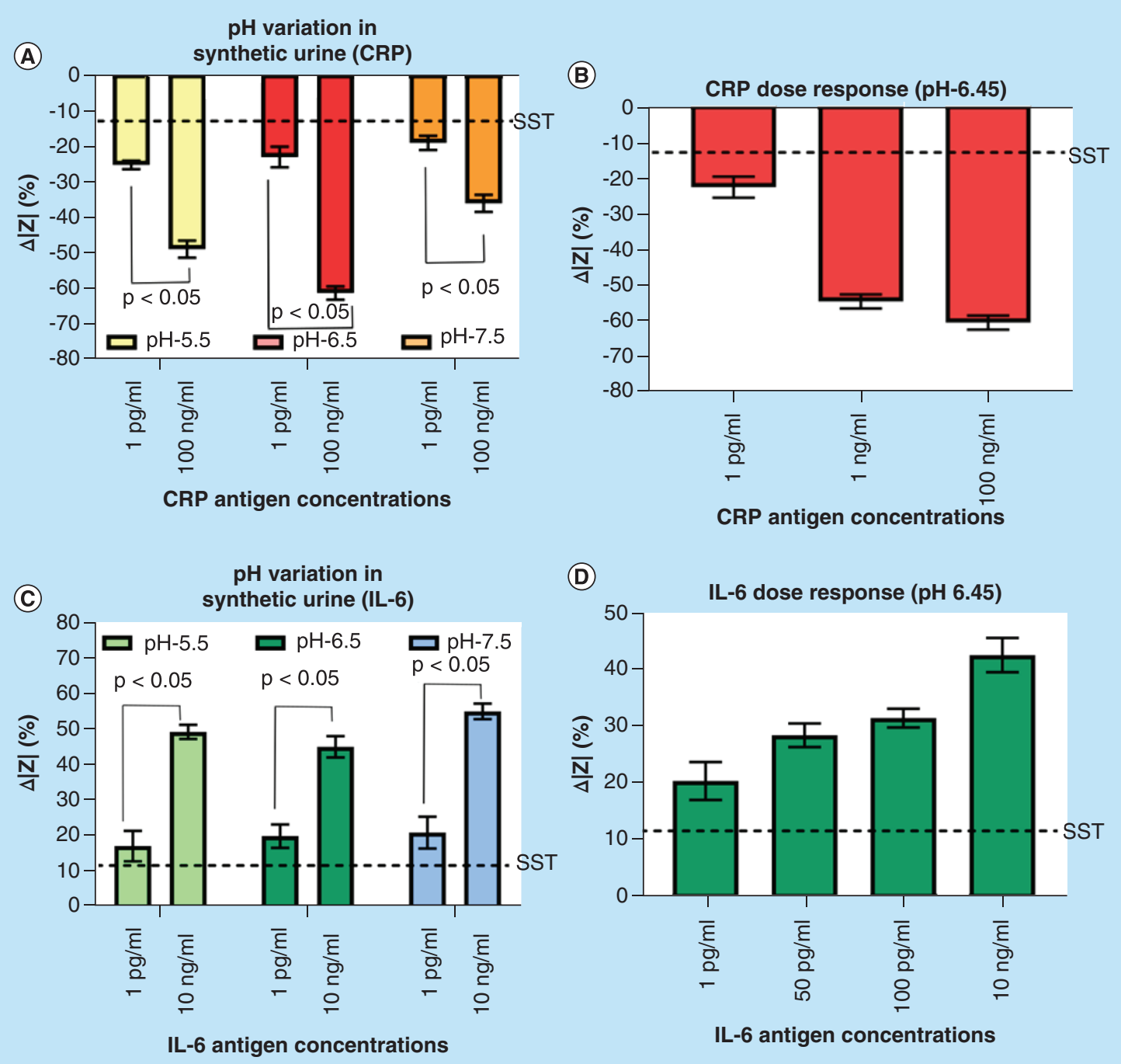

IL-6 antigen concentrations

Figure 3. Electrochemical impedance spectroscopy characterization of CRP and IL-6 in synthetic urine. (A) Change in impedance analysis for low and high CRP antigen concentrations in three $\mathrm{pH}$ ranges of synthetic urine. Dotted lines indicate SST; (B) Impedance analysis for pH 6.45 synthetic urine; (C) Change in impedance analysis for low and high IL- 6 antigen concentrations in three $\mathrm{pH}$ buffer ranges of synthetic urine. The dotted lines indicate SST; (D) Change in impedance analysis for four IL- 6 antigen concentrations in pH 6.45 buffer of synthetic urine. CRP: C-reactive protein; SST: Specific signal threshold.

using $t$-test for all three $\mathrm{pH}$ buffers of the synthetic urine, thereby indicating the sensitivity of Mo electrode to detect CRP in varying ionic conditions of urine. Figure $3 \mathrm{~B}$ shows the calibration dose response for CRP using synthetic urine at $\mathrm{pH}$ buffer 6.45 , similar to the $\mathrm{pH}$ of pooled human samples as described in section 4.4.1. The change in impedance ranged from approximately $-22 \%$ for $1 \mathrm{pg} / \mathrm{ml}$ CRP concentration to approximately $-61 \%$ for $100 \mathrm{ng} / \mathrm{ml} \mathrm{CRP}$ concentration, and was governed by the equation: $\Delta|\mathrm{Z}|=-3.451^{*} \ln [\mathrm{CRP}$ in $\mathrm{ng} / \mathrm{ml}]-48.82$ demonstrating an $\mathrm{R}^{2}$ value of 0.932 . A specific signal threshold (SST) of $13 \%$ was computed using the following formula.

$$
\mathrm{SST}=\left((3 * \mathrm{SD}) / \mathrm{Z}_{\text {Baseline }}\right) * 100
$$

A limit of detection (LOD) of $1 \mathrm{pg} / \mathrm{ml}$ was obtained which is the smallest concentration above SST. Figure 3C shows the percent change in impedance at low $(1 \mathrm{pg} / \mathrm{ml})$ and high $(10 \mathrm{ng} / \mathrm{ml})$ concentrations of IL- 6 across varying 


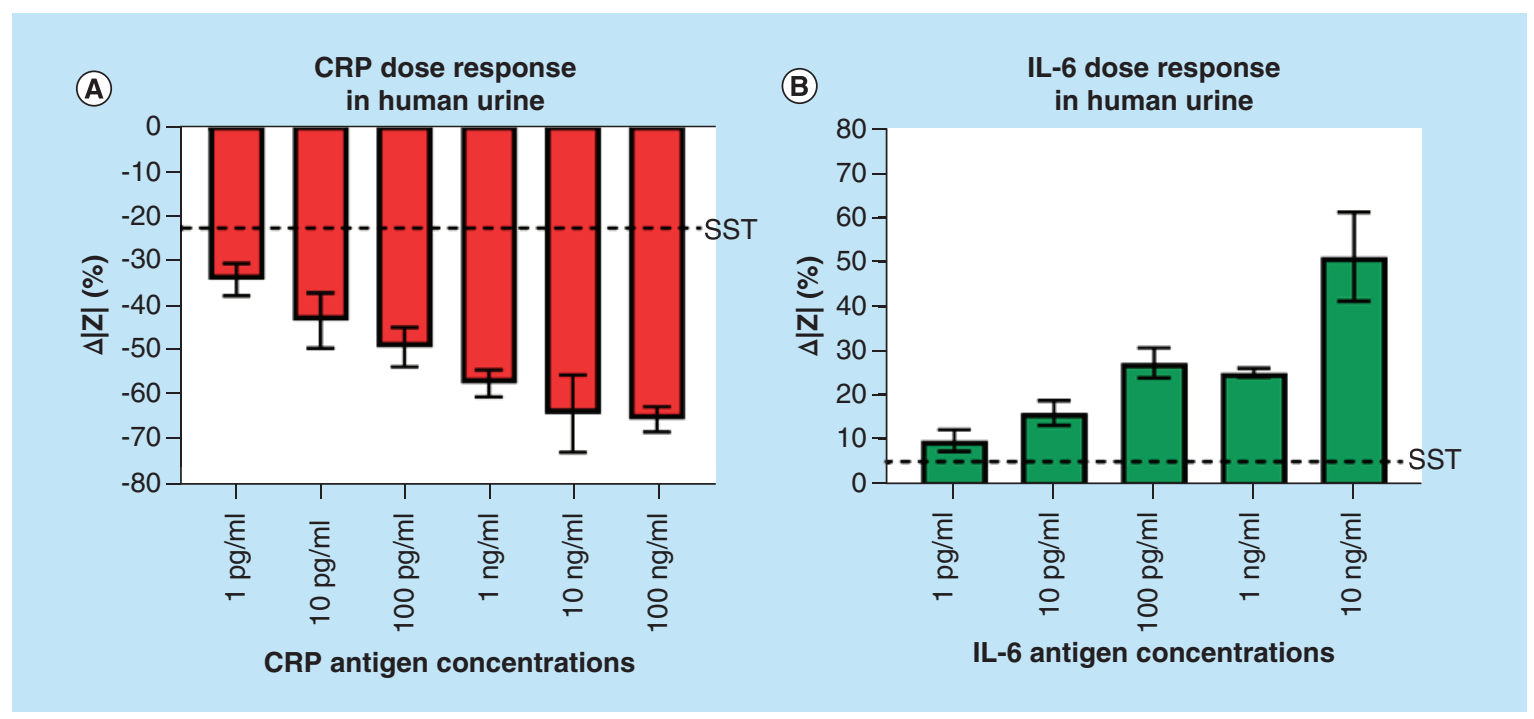

Figure 4. Electrochemical impedance spectroscopy characterization of CRP and IL-6 in human urine. (A) Change in impedance analysis for CRP antigen concentrations; (B) Change in impedance analysis for IL-6 antigen concentrations. Dotted lines indicate SST.

CRP: C-reactive protein; SST: Specific signal threshold.

$\mathrm{pH}$. The percentage change in impedance for $1 \mathrm{pg} / \mathrm{ml}$ of IL-6 antigen was calculated to be approximately $20 \%$ and approximately $50 \%$ for $10 \mathrm{ng} / \mathrm{ml}$ at all three $\mathrm{pH}$ ranges. This presents an opposing trend to the impedance response observed in Figure 3A and B for CRP, which is in agreement with the $\zeta$-potential data presented in Figure 2. Figure 3D shows the calibration dosing response for $1 \mathrm{pg} / \mathrm{ml}$ to $10 \mathrm{ng} / \mathrm{ml} \mathrm{IL}-6 \mathrm{in} \mathrm{pH} 6.45$ synthetic urine, and was governed by the equation: $\Delta|\mathrm{Z}|=2.405 * \ln [\mathrm{IL}-6 \mathrm{in} \mathrm{ng} / \mathrm{ml}]+36.20$ demonstrating an $\mathrm{R}^{2}$ value of 0.995 . All dose responses were above the measured SST of $11 \%$ with LOD of $1 \mathrm{pg} / \mathrm{ml}$. These results help to establish that the sensor is robust to varying $\mathrm{pH}$ and is suitable for testing in physiological buffer. The stability of the antibody functionalization on sensor surface was established through multiple buffer wash steps post Ab functionalization. The impedance values of the subsequent buffer wash steps were statistically insignificant $(p>0.05)$. The plot has been shown in Supplementary Figure 2A. The specificity of the assay was demonstrated with the addition of creatinine on an IL- 6 antibody-functionalized sensor and the results are included in the supplementary information (Supplementary Figure 2B). The absence of correlation between the changes in the impedance with increasing creatinine concentrations spanning its physiologically expressed range established the specificity of the developed assay.

\section{EIS characterization of CRP \& IL-6 in human urine}

After establishing the sensor's performance in synthetic urine, the sensor was tested using spiked samples of IL-6 and CRP in pooled human urine to evaluate the electrochemical response of the biosensor, and to demonstrate translatability of the technology to complex human samples.

Figure $4 \mathrm{~A}$ indicates the percentage change in impedance for CRP antigen concentrations from $1 \mathrm{pg} / \mathrm{ml}$ to $100 \mathrm{ng} / \mathrm{ml}$. An SST of 21\% was calculated for the CRP detection assay. The LOD was therefore determined to be at $1 \mathrm{pg} / \mathrm{ml}$, as it is still detected over the established SST. The LOD is within the normal levels of CRP observed in urine. The change in impedance ranges from -34 to $-65 \%$ increasing as a function of $\mathrm{C}_{\mathrm{dl}}$ modulation with increasing CRP concentrations. Figure $4 \mathrm{~B}$ shows the change in impedance for the IL- 6 antigen concentrations from $1 \mathrm{pg} / \mathrm{ml}$ to $10 \mathrm{ng} / \mathrm{ml}$. The LOD for the IL- 6 detection was also determined to be $1 \mathrm{pg} / \mathrm{ml}$, which is in the normal physiological range of IL-6 in urine. The change in impedance ranged from 10 to $51 \%$ and is above the $5 \%$ SST established for this study. The governing equation for the linear dynamic range from $1 \mathrm{pg} / \mathrm{ml}$ to $10 \mathrm{ng} / \mathrm{ml}$ is: $\Delta|\mathrm{Z}|=3.970^{*}(\mathrm{IL}-6$ in $\mathrm{ng} / \mathrm{ml})+34.95$ demonstrating an $\mathrm{R}^{2}$ value of 0.844 . 

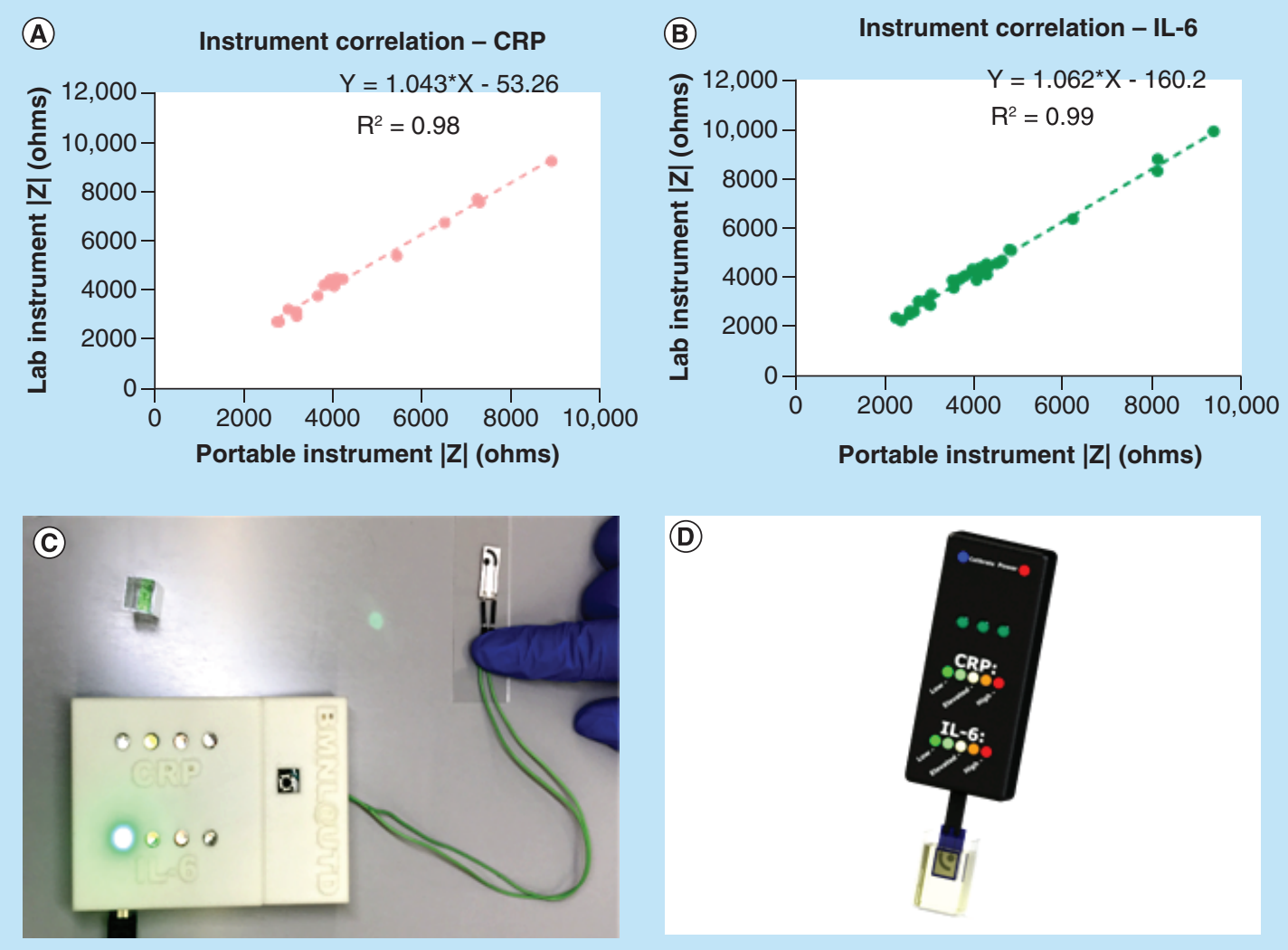

Figure 5. Performance of portable electronic device. (A) Impedance correlation between lab instrument and portable instrument for CRP; (B) Impedance correlation between lab instrument and portable instrument for IL-6; (C) Visual demonstration of test results using electronic interface; (D) 3D model representation of portable urine dipstick probe interface.

CRP: C-reactive protein.

\section{Comparison of impedance response with portable electronic hardware prototype}

To demonstrate the translatability of the developed dipstick probe assay into a true portable point-of-care device, a portable reader was developed. The device was designed to perform single frequency EIS measurements at the established $1 \mathrm{~Hz}$ analysis point used for detection of the target proteins on the benchtop instrument. The device was designed to capture the changes in impedance response of the sensor from the postfunctionalization baseline to the selected dose regimes of either CRP or IL- 6 to the same level of sensitivity as can be attained by the benchtop instrument.

To quantifiably establish comparability of the device's measurements to the benchtop instrument, the raw impedance values of the device were plotted against the corresponding impedance values of the benchtop instrument for the detection of both molecules. This correlation is plotted for CRP in Figure 5A, showing a linear response with an $R^{2}$ value of 0.98 with a slope near 1.0. The governing equation can be defined as $y=1.043 x-53.26$. Figure 5B shows the same correlation when detecting IL-6, showing a linear response with an $\mathrm{R}^{2}$ value of 0.99 with a slope near 1.0. The governing equation can be defined as $y=1.062 \mathrm{x}-160.20$. These results show that the device is sensitive to the range of impedances produced by the sensor showing comparable performance to the benchtop instrument. Figure 5C shows the physical device and demonstrates an example of reporting elevated levels of IL-6 $(1-100 \mathrm{pg} / \mathrm{ml})$, while Figure 5D shows the envisioned commercial implementation of this device.

\section{Discussion}

The surface characterization experimental results established that there is a uniform and smooth deposition of Mo on the nanoporous polyamide substrate. The FTIR analysis of the blank Mo substrate indicated the presence of 
a passive oxide layer which is leveraged for binding of EDC-NHS crosslinker molecules to the electrode surface. After EDC-NHS binding, the peak seen at $1650 \mathrm{~cm}^{-1}$ is indicative of the $\mathrm{v}(\mathrm{C}=\mathrm{O})$ carbonyl stretching of the EDC-NHS. The carbonyl stretching is expected postantibody conjugation and is observed in both antibody measurements (1647 and $1656 \mathrm{~cm}^{-1}$ for IL-6 and CRP antibody, respectively). The peak at $1566 \mathrm{~cm}^{-1}$ observed in the EDC-NHS measurement is from $\mathrm{v}_{\mathrm{as}}(\mathrm{N}-\mathrm{O})$ nitro stretching of the crosslinker, which disappears in the antibody measurements after the aminolysis of the NHS after binding. Once the antibody binds, a new bond $\mathrm{v}_{\mathrm{as}}(\mathrm{N}-\mathrm{C}, \mathrm{C}-\mathrm{C})$ can be seen at 1082 and $1047 \mathrm{~cm}^{-1}$ for IL-6 and CRP antibody, respectively. This evidence suggests that EDC-NHS has strongly bound to the Mo oxide layer, and is stable through the functionalization steps associated with the assay. The results also indicate that both the IL-6 and CRP antibodies are binding to the EDC-NHS crosslinker at the surface of the Mo as opposed to other mechanisms such as physical absorption.

The $\zeta$-potential measurements indicate the surface charge changes and hence we leveraged this property toward characterizing the modulation of the EDL due to antibody-antigen binding of IL- 6 and CRP on the Mo nanoparticle surface. $\zeta$-potential is also a measure of repulsion behavior of identical charged species. As a larger molecule $(105 \mathrm{kDa})$, the number of identically charged species is greater at higher dose concentrations of CRP, thus, the $\zeta$-potential increases with increasing dose concentrations. Since, IL- 6 is relatively small based on molecular weight $(26 \mathrm{kDa})$, the number of IL- 6 molecules docking on to individual Mo surface would be higher at higher concentration. This results in a lower surface charge, thus, reducing the $\zeta$-potential at higher concentrations. The opposing trends in $\zeta$-potential with respect to concentrations of IL- 6 and CRP are due to the difference in the binding mechanism of both the biomolecules on the Mo surface, thus, modulating the EDL differently. EDL consists of two layers, in other words, stern layer and diffuse layer. There exists a 'slipping plane' within the diffuse layer where the potential drops linearly from the stern plane. Beyond the slipping plane, the potential drops exponentially. The potential at the slipping plane is the $\zeta$-potential. Higher molecular weight of the macromolecular species results in the slipping plane at a larger distance from the stern plane [43]. The higher $\zeta$-potential of CRP as compared with IL- 6 can thus be attributed to the higher molecular weight of CRP. As $\zeta$-potential is a measure of $\mathrm{EDL}$, it can be used to correlate with techniques that modulate the EDL with small voltage perturbations [41].

The changes in impedance due to biomolecular binding of CRP and IL-6 to the corresponding capture probes at the electrode/solution interface were analyzed through EIS. The primary objective was to evaluate the electrochemical response of the developed biosensor across varying $\mathrm{pH}$ of urine. Nonfaradaic EIS primarily captures the EDL dynamics at the electrode-solution interface due to small voltage perturbations. The effect of solution resistance is a surface phenomenon and is typically dominated at higher frequencies of the impedance spectrum [44]. The change in impedance within the EDL is mainly due to modulation of the $\mathrm{C}_{\mathrm{dl}}$ resulting from the binding between specific antibody and antigen interaction. The impedance response with varying concentrations of the target analyte were analyzed at $1 \mathrm{~Hz}$ frequency due to the dominance of $\mathrm{C}_{\mathrm{dl}}$ at low frequencies [44,45]. Also, maximum change in impedance response for multiple antigen concentrations was observed at $1 \mathrm{~Hz}$ frequency and hence it was chosen as the frequency of analysis of impedance spectra. The negative change in impedance for the CRP dose concentrations indicates that the impedance of dose concentration increases from the baseline impedance. This phenomenon can be attributed to the charge state of CRP in the urine buffer. The dipstick probe biosensor exhibits a wide dynamic range that spans over normal and elevated levels for both CRP and IL-6 in human urine samples, thereby establishing its usefulness to be used as an electronic dipstick probe biosensor.

The goal of the portable electronic reader was to establish that the device was sensitive to the range of impedances exhibited by the sensor, and that the device reports those impedance values with the same accuracy as the benchtop instrument. The main source of error in the correlation of the two tools came from fluctuations in solution resistance as function of time when reconfiguring the sensor from one tool to another, which is not a factor of the device itself. Overall, the device demonstrates translatability of the urinary dipstick for inflammatory biomarkers toward a true point-of-care diagnostic form factor.

\section{Conclusion}

An affinity-based electrochemical biosensor for the detection of two critical inflammatory biomarkers, namely CRP and IL-6, was developed using Mo electrode on a nanoporous flexible substrate. The surface characterization performed through SEM analysis demonstrated a uniform deposition of Mo on the polyamide substrate. The presence of peaks, in FTIR spectra, correlated with the EDC-NHS crosslinker molecules, thereby establishing the surface functionalization of Mo electrode with crosslinker monolayer. Binding of CRP and IL-6 antibody with EDC-NHS-functionalized Mo surface was confirmed using FTIR characterization. The change in the 
surface charge, calculated through $\zeta$-potential measurements, correlated to the antigen concentrations, thereby validating antigen binding to crosslinker-antibody conjugate. The affinity-based antigen-antibody binding was characterized through nonfaradaic EIS. The dipstick probe biosensor demonstrated ability to detect CRP and IL-6 antigen concentration as low as $1 \mathrm{pg} / \mathrm{ml}$ in both pooled human urine and synthetic urine samples. The detection concentration range correlated with threshold cut-off concentrations of diseases associated with these biomarkers. The sensor's performance was preserved in multiple $\mathrm{pH}$ range of synthetic urine. This work is the first demonstration of combinatorial detection of CRP and IL- 6 in urine using an electrochemical dipstick probe with Mo electrode. The portable research interface established a close correlation with benchtop measurements thereby supporting its development as a semiquantitative point of care biosensor for near-patient testing for detecting inflammatory biomarkers.

\section{Future perspective}

The current Mo electrode nanoporous dipstick probe biosensor has the capability to be developed as a quantitative error-free reporting platform for biomarker analysis in human urine. The biosensor holds potential for detecting multiple biomarkers from a single sample. The portable electronic hardware is envisioned as a point-of-care diagnostics tool to be used in clinics and in a home environment. The stability of the electrode enables dynamic monitoring of urinary biomarkers over multiple time intervals thereby benefiting patients with chronic health conditions.

\section{Summary points}

- Label-free electrochemical urine dipstick probe with molybdenum electrode has been developed for combinatorial detection of IL-6 and C-reactive protein in urine.

- The performance of the electrode to detect the target biomarkers has been evaluated in synthetic urine with multiple $\mathrm{pH}$ buffers and in human urine.

- The feasibility to perform point-of-care detection in urine has been demonstrated with a portable electronic research hardware.

- Distinguishability between normal and elevated levels of C-reactive protein and IL-6 in urine has been demonstrated.

\section{Supplementary data}

To view the supplementary data that accompany this paper please visit the journal website at: www.future-science.com/doi/suppl /10.4155/fsoa-2017-0142.

\section{Author contributions}

V Kamakoti, K Hyeok Choi, B Jagannath and D Kinnamon performed the experiments. V Kamakoti, B Jagannath, D Kinnamon and S Prasad wrote the manuscript.

\section{Financial \& competing interests disclosure}

The authors have no relevant affiliationsor financial involvement with any organization or entity with a financial interest in or financial conflict with the subject matter or materials discussed in the manuscript. This includes employment, consultancies, honoraria, stock ownership or options, expert testimony, grants or patents received or pending, or royalties.

No writing assistance was utilized in the production of this manuscript.

\section{Acknowledgements}

The authors thank KC Lin for helping with atomic force microscopy characterization experiments, J Craven for helping with scanning electron microscope characterization and NR Shanmugam for helping with energy dispersive $\mathrm{x}$-ray spectroscopy characterization experiments.

\section{Ethical conduct of research}

The authors state that they have obtained appropriate institutional review board approval or have followed the principles outlined in the Declaration of Helsinki for all human or animal experimental investigations. In addition, for investigations involving human subjects, informed consent has been obtained from the participants involved. 
Open access

This work is licensed under the Creative Commons Attribution 4.0 License. To view a copy of this license, visit http://creativecomm ons.org/licenses/by/4.0/

\section{References}

Papers of special note have been highlighted as: $\bullet$ of interest; $\bullet \bullet$ of considerable interest

1. Prasad S, Tyagi AK, Aggarwal BB. Detection of inflammatory biomarkers in saliva and urine: potential in diagnosis, prevention and treatment for chronic diseases. Exp. Biol. Med. 241(8), 783-799 (2016).

-• Presents a summary of major inflammatory biomarkers in saliva and urine.

2. Kamakoti V. Evaluation of molybdenum as an electrode material for affinity-based urine dipstick biosensing (2017). http://libtreasures.utdallas.edu/xmlui/handle/10735.1/5482

3. Chen Z, Martin MT, Xinyu L. A microfluidic paper-based electrochemical biosensor array for multiplexed detection of metabolic biomarkers. Sci. Technol. Adv. Mater. 14(5), 054402 (2013).

4. Link N, Weber W, Fussenegger M. A novel generic dipstick-based technology for rapid and precise detection of tetracycline, streptogramin and macrolide antibiotics in food samples. J. Biotechnol. 128(3), 668-680 (2007).

5. Selvakumar LS, Thakur MS. Dipstick-based immunochemiluminescence biosensor for the analysis of vitamin B12 in energy drinks: a novel approach. Anal. Chim. Acta 722, 107-113 (2012).

6. Vlachou MA, Glynou KM, Ioannou PC, Christopoulos TK, Vartholomatos G. Development of a three-biosensor panel for the visual detection of thrombophilia-associated mutations. Biosens. Bioelectron. 26(1), 228-234 (2010).

7. Peter J, Green C, Hoelscher M, Mwaba P, Zumla A, Dheda K. Urine for the diagnosis of tuberculosis: current approaches, clinical applicability and new developments. Curr. Opin. Pulm. Med. 16(3), 262 (2010).

8. Gourinat A-C, O'connor O, Calvez E, Goarant C, Dupont-Rouzeyrol M. Detection of Zika virus in urine. Emerging Infect. Dis. 21(1), 84 (2015).

9. Diouf A, Motia S, Hassani NEaE, Bari NE, Bouchikhi B. An electrochemical biosensor-based on moleculary imprinted polymer for the quantification of creatinine in human urine samples. Presented at: 2017 ISOCS/IEEE International Symposium on Olfaction and Electronic Nose (ISOEN). Montreal, Canada, 28-31 May 2017.

10. He Y, Zhang X, Yu H. Gold nanoparticles-based colorimetric and visual creatinine assay. Microchimica Acta 182(11), 2037-2043 (2015).

11. Su L, Feng J, Zhou X, Ren C, Li H, Chen X. Colorimetric detection of urine glucose-based ZnFe2O4 magnetic nanoparticles. Anal. Chem. 84(13), 5753-5758 (2012).

12. Zangheri M, Di Nardo F, Mirasoli M et al. Chemiluminescence lateral flow immunoassay cartridge with integrated amorphous silicon photosensors array for human serum albumin detection in urine samples. Anal. Bioanal. Chem. 408(30), 8869-8879 (2016).

13. González-Guerrero AB, Maldonado J, Dante S, Grajales D, Lechuga LM. Direct and label-free detection of the human growth hormone in urine by an ultrasensitive bimodal waveguide biosensor. J. Biophotonics 10(1), 61-67 (2017).

14. Chen C-H, Lin MS. A novel structural specific creatinine sensing scheme for the determination of the urine creatinine. Biosens. Bioelectron. 31(1), 90-94 (2012).

15. Mach KE, Mohan R, Patel S, Wong PK, Hsieh M, Liao JC. Development of a biosensor-based rapid urine test for detection of urogenital schistosomiasis. PLoS Negl. Trop. Dis. 9(7), e0003845 (2015).

16. Mukundan H, Kumar S, Price DN et al. Rapid detection of mycobacterium tuberculosis biomarkers in a sandwich immunoassay format using a waveguide-based optical biosensor. Tuberculosis 92(5), 407-416 (2012).

17. Cho S, Park TS, Nahapetian TG, Yoon J-Y. Smartphone-based, sensitive $\mu$ PAD detection of urinary tract infection and gonorrhea. Biosens. Bioelectron. 74, 601-611 (2015).

18. Li N, Wang Y, Li Y et al. A label-free electrochemical immunosensor based on Au@Pd/Ag yolk-bimetallic shell nanoparticles and amination graphene for detection of nuclear matrix protein 22. Sens Actuators B Chem. 202, 67-73 (2014).

19. Xuan Viet N, Chikae M, Ukita Y et al. Gold-linked electrochemical immunoassay on single-walled carbon nanotube for highly sensitive detection of human chorionic gonadotropinhormone. Biosens. Bioelectron. 42, 592-597 (2013).

20. Sánchez-Tirado E, Martínez-García G, González-Cortés A, Yáñez-Sedeño P, Pingarrón JM. Electrochemical immunosensor for sensitive determination of transforming growth factor (TGF)- $\beta 1$ in urine. Biosens. Bioelectron. 88, 9-14 (2017).

21. Pan Y, Sonn GA, Sin MLY et al. Electrochemical immunosensor detection of urinary lactoferrin in clinical samples for urinary tract infection diagnosis. Biosens. Bioelectron. 26(2), 649-654 (2010).

22. Park Y-J, Yoo S-A, Kim G-R, Cho C-S, Kim W-U. Urinary interleukin-6 as a predictor of radiographic progression in rheumatoid arthritis: a 3-year evaluation. Sci. Rep. 6, 35242 (2016).

23. Krzemień G, Roszkowska-Blaim M, Kostro I et al. Urinary levels of interleukin- 6 and interleukin- 8 in children with urinary tract infections to age 2. Med. Sci. Monit. 10(11), CR593-CR597 (2004). 
24. Gürgöze MK, Akarsu S, Yilmaz E et al. Proinflammatory cytokines and procalcitonin in children with acute pyelonephritis. Pediatr. Nephrol. 20(10), 1445-1448 (2005).

25. Galanakis E, Bitsori M, Dimitriou H, Giannakopoulou C, Karkavitsas NS, Kalmanti M. Serum and urine interleukin-6 and transforming growth factor-beta1 in young infants with pyelonephritis. Int. Urol. Nephrol. 39(2), 581-585 (2007).

26. Freedman AL. Urologic diseases in North America project: trends in resource utilization for urinary tract infections in children. $J$. Urol. 173(3), 949-954 (2005).

27. Griebling TL. Urologic diseases in America project: trends in resource use for urinary tract infections in women. J. Urol. 173(4), 1281-1287 (2005).

28. Krzemień G, Szmigielska A, Turczyn A, Pańczyk-Tomaszewska M. Urine interleukin-6, interleukin-8 and transforming growth factor $\beta 1$ in infants with urinary tract infection and asymptomatic bacteriuria. Cent. Eur. J. Immunol. 41(3), 260 (2016).

- Explains the association of IL-6 with urinary tract infections.

29. Uehling DT, Johnson DB, Hopkins WJ. The urinary tract response to entry of pathogens. World J. Urol. 17(6), 351-358 (1999).

30. Sundvall P-D, Elm M, Ulleryd P et al. Interleukin- 6 concentrations in the urine and dipstick analyses were related to bacteriuria but not symptoms in the elderly: a cross-sectional study of 421 nursing home residents. BMC Geriatr. 14(1), 88 (2014).

31. Sundén F, Wullt B. Predictive value of urinary interleukin-6 for symptomatic urinary tract infections in a nursing home population. Int. J. Urol. 23(2), 168-174 (2016).

32. Tejani NR, Chonmaitree T, Rassin DK, Howie VM, Owen MJ, Goldman AS. Use of C-reactive protein in differentiation between acute bacterial and viral otitis media. Pediatrics 95(5), 664-669 (1995).

33. Chuang Y-C, Tyagi V, Liu R-T, Chancellor MB, Tyagi P. Urine and serum C-reactive protein levels as potential biomarkers of lower urinary tract symptoms. Urol. Sci. 21(3), 132-136 (2010).

34. Meyer MH, Hartmann M, Krause H-J et al. CRP determination based on a novel magnetic biosensor. Biosens. Bioelectron. 22(6), 973-979 (2007).

35. Yildiz B, Poyraz H, Cetin N, Kural N, Colak O. High sensitive C-reactive protein: a new marker for urinary tract infection, VUR and renal scar. Eur. Rev. Med. Pharmacol. Sci. 17, 2598-2604 (2013)

36. Nanda N, Juthani-Mehta M. Novel biomarkers for the diagnosis of urinary tract infection: a systematic review. Biomark. Insights 4 , $111-121(2009)$

37. Kamakoti V, Panneer Selvam A, Radha Shanmugam N, Muthukumar S, Prasad S. Flexible molybdenum electrodes toward designing affinity-based protein biosensors. Biosensors 6(3), 36 (2016).

38. Munje RD, Muthukumar S, Selvam AP, Prasad S. Flexible nanoporous tunable electrical double layer biosensors for sweat diagnostics. Sci. Rep. 5, 14586 (2015).

39. Panneer Selvam A, Prasad S, Barrett TW, Kazmierczak SC. Electrical nanowell diagnostics sensors for rapid and ultrasensitive detection of prostate-specific antigen. Nanomedicine 10(16), 2527-2536 (2015).

- Provides the explanation on signal enhancements due to the nanoporous structures.

40. Radha Shanmugam N, Muthukumar S, Chaudhry S, Anguiano J, Prasad S. Ultrasensitive nanostructure sensor arrays on flexible substrates for multiplexed and simultaneous electrochemical detection of a panel of cardiac biomarkers. Biosens. Bioelectron. 89, 764-772 (2017).

41. Munje RD, Muthukumar S, Jagannath B, Prasad S. A new paradigm in sweat-based wearable diagnostics biosensors using room temperature ionic liquids (RTILs). Sci. Rep. 7, 1950 (2017).

-. Describes the correlation between surface charge characterization and modulation of electrical double layer.

42. Xu K, Wang C, Liu H, Qian Y. Simultaneous removal of phosphorus and potassium from synthetic urine through the precipitation of magnesium potassium phosphate hexahydrate. Chemosphere 84(2), 207-212 (2011).

43. Lin K-C, Jagannath B, Muthukumar S, Prasad S. Subpicomolar label-free detection of thrombin using electrochemical impedance spectroscopy of aptamer-functionalized MoS2. Analyst 142(15), 2770-2780 (2017).

44. Daniels JS, Pourmand N. Label-free impedance biosensors: Opportunities and challenges. Electroanalysis 19(12), 1239-1257 (2007).

-• Provides an explanation of framework for electrial-circuit-level modeling of nonfaradaic biosensor system components.

45. Munje RD, Muthukumar S, Prasad S. Lancet-free and label-free diagnostics of glucose in sweat using zinc oxide-based flexible bioelectronics. Sens Actuators B Chem. 238, 482-490 (2017) 